\title{
Hyperons in thermal QCD from the lattice*
}

\author{
Gert Aarts ${ }^{1}$, Chris Allton ${ }^{1 \dagger}$, Davide de Boni ${ }^{1}$, Jonas Glesaaen ${ }^{1}$, Simon Hands ${ }^{1}$, \\ Benjamin Jger ${ }^{2}$, and Jon-Ivar Skullerud ${ }^{3}$ \\ 1 Department of Physics, College of Science, Swansea University, Swansea SA2 8PP, \\ United Kingdom \\ ${ }^{2} \mathrm{CP}^{3}$-Origins \& Danish IAS, Department of Mathematics and Computer Science, \\ University of Southern Denmark, 5230 Odense M, Denmark \\ 3 Department of Theoretical Physics, National University of Ireland Maynooth, \\ Maynooth, County Kildare, Ireland \\ †Speaker c.allton@swansea.ac.uk
}

\begin{abstract}
We study the spectrum of light baryons and hyperons as a function of temperature using lattice gauge theory methods. We find that masses of positive parity states are temperature independent, within errors, in the hadronic phase. The negative parity states decrease in mass as the temperature increases. Above the deconfining temperature, lattice correlators and spectral functions show a degeneracy between parity sectors, i.e. parity doubling. We apply our findings to an in-medium Hadron Resonance Gas model. The techniques used in this study include direct analysis of the hadronic correlation functions, conventional fitting procedures, and the Maximum Entropy Method.
\end{abstract}

Keywords: QCD spectrum, thermal QCD, Lattice gauge theory

\section{Introduction}

Symmetries play a crucial role in the Standard Model and are especially significant in the QCD transition from the confining, chirally broken, hadronic phase to the deconfined, chirally symmetric, plasma phase. Chiral symmetry restoration in the meson sector at finite temperature has been studied extensively, but there have been very few studies in baryons $[1 / 2] 3$.

In the baryonic case, a combination of unbroken chiral symmetry and parity leads to parity doubling, i.e. a degeneracy between positive and negative parity states. Hence it is expected that in the chirally symmetric phase, baryonic channels related by parity will display approximate degeneracy. This work tests this conjecture by studying octet and decuplet light baryons and hyperons as the temperature varies using our FASTSUM Collaboration's anisotropic, $2+1$ flavour lattices [4].

We find evidence of parity doubling above the deconfining temperature, $T_{c}{ }_{4}^{4}$ with this degeneracy being most pronounced for the baryons with the smallest

\footnotetext{
${ }^{4} T_{c}$ is not uniquely defined. Here it's defined from the renormalised Polyakov loop 4 .

* Invited plenary talk presented at the Strangeness in Quark Matter Conference (SQM 2019), Bari, Italy, 10-15 June 2019.
} 
strange quark content. In the hadronic phase we find that the negative parity masses decrease as $T \rightarrow T_{c}$ while the corresponding positive parity masses remain constant within the uncertainty. We use these temperature-dependent masses to define an in-medium Hadron Resonance Gas, deriving results for susceptibilities and partial pressures.

The work presented here is detailed more fully in 5677 .

\section{Parity in Baryons}

We use the standard interpolation operator for a nucleon

$$
O_{N}(\mathbf{x}, \tau)=\epsilon_{a b c} u_{a}(\mathbf{x}, \tau)\left[u_{b}^{T}(\mathbf{x}, \tau) \mathcal{C} \gamma_{5} d_{c}(\mathbf{x}, \tau)\right]
$$

where $u, d$ are the quark fields, $a, b, c$ are colour indices, other indices are suppressed and $\mathcal{C}$ denotes the charge conjugation matrix. Similar operators are used for octet and decuplet cases, see [6. Parity operators for the positive and negative parity channels are defined as $O_{N_{ \pm}}(\mathbf{x}, \tau)=P_{ \pm} O_{N}(\mathbf{x}, \tau)$, where $P_{ \pm}=\frac{1}{2}\left(1 \pm \gamma_{4}\right)$. We study the usual Euclidean correlators of these operators, summed over the Dirac indices and projected to zero momentum,

$$
G_{ \pm}(\tau)=\int d^{3} x\left\langle O_{N_{ \pm}}(\mathbf{x}, \tau) \bar{O}_{N_{ \pm}}(\mathbf{0}, 0)\right\rangle .
$$

From the properties of Euclidean time reflection, it follows that the forward (backward) propagation of $G_{+}(\tau)$ corresponds to the positive (negative) parity channel (see e.g. [5]). Hence both parities are obtained from one correlator.

\section{$3 \quad$ Lattice parameters}

Our FASTSUM collaboration specialises in using a fixed-scale approach on anisotropic lattices where the temporal lattice spacing $a_{\tau}$ is smaller than the spatial one $a_{s}$. We use $2+1$ quark flavours, where the strange quark mass has its physical value, but the two lightest flavours are heavier than in nature resulting in a pion mass of $M_{\pi}=392(4) \mathrm{MeV}$. The inverse temporal lattice spacing is $a_{\tau}^{-1}=$ $5.63(4) \mathrm{GeV}$ with $a_{s} / a_{\tau} \approx 3.5$, and our spatial lattice volume is $24^{3}$. We use a variety of temporal extents, $N_{\tau}$, with the corresponding temperatures $T=$ $1 /\left(a_{\tau} N_{\tau}\right)$ shown in Table 1 s spanning both phases.

\begin{tabular}{l|rrrrrrrr}
\hline$N_{\tau}$ & 128 & 40 & 36 & 32 & 28 & 24 & 20 & 16 \\
$T / T_{c}$ & 0.24 & 0.76 & 0.84 & 0.95 & 1.09 & 1.27 & 1.52 & 1.90 \\
$T[\mathrm{MeV}]$ & 44 & 141 & 156 & 176 & 201 & 235 & 281 & 352 \\
\hline
\end{tabular}

Table 1. Lattice parameters and temperatures studied. The ensemble at the lowest temperature was provided by the HadSpec Collaboration 8 ] 


\section{Results}

The nucleon correlation functions are shown in Fig. 1 for both parity states. Other channels have similar behaviour. The backward movers (from the second half of the temporal range) have been reflected in $\tau$ to enable direct comparison with their positive counterparts.

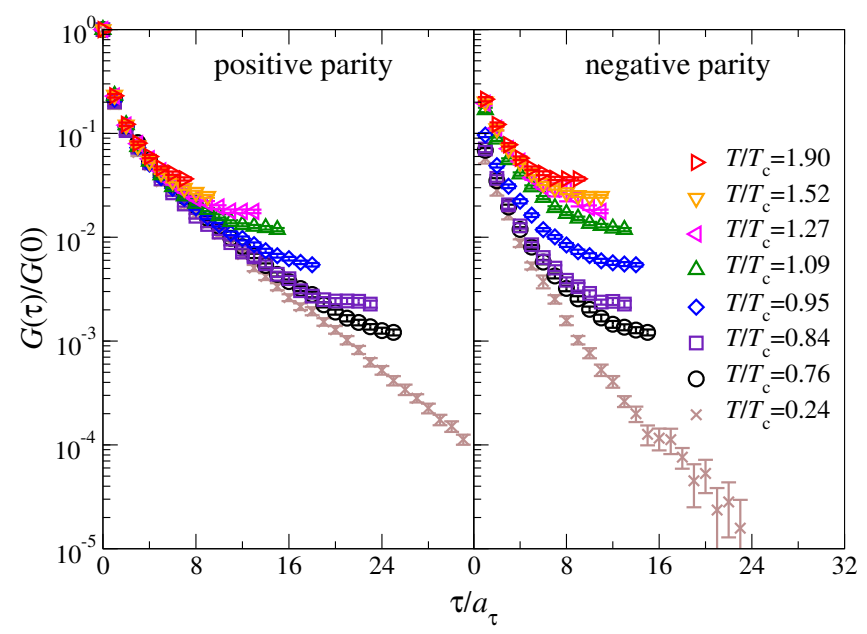

Fig. 1. Temperature dependent correlators for the + ve and -ve parity nucleon channels.
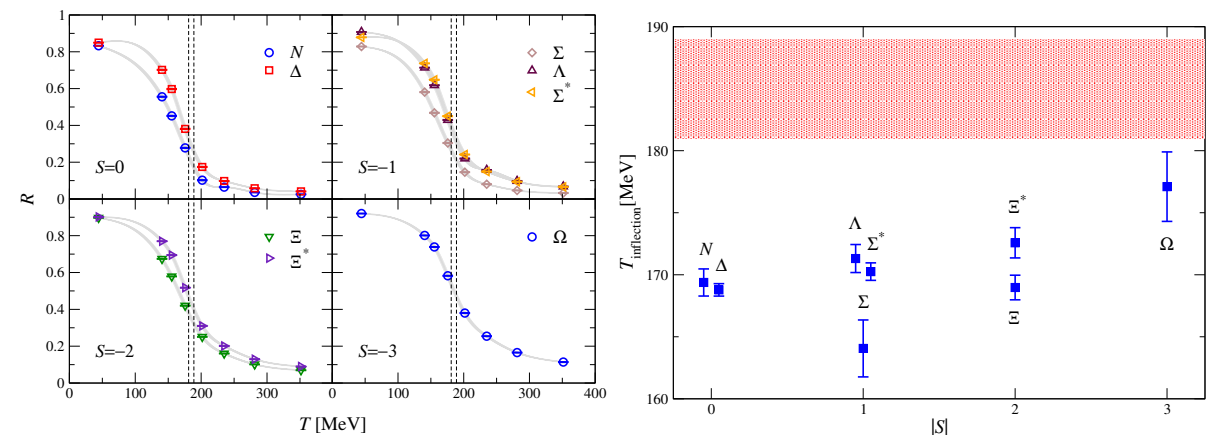

Fig. 2. (Left) The ratio $R$ for various strangeness sectors. (Right) Inflection point temperature of $R$ with $T_{c}$ from the Polyakov loop shown in the band [4.

We define the ratio $R(\tau)=\left[G_{+}(\tau)-G_{+}(1 / T-\tau)\right] /\left[G_{+}(\tau)+G_{+}(1 / T-\tau)\right]$ which measures the time reflection (a)symmetry of $G_{+}(\tau)$ and therefore the 
presence (absence) of parity doubling. $R(\tau) \sim 1$ signifies non-degenerate parity states, and $R(\tau) \sim 0$ parity doubling. It is convenient to average $R(\tau)$ over $\tau$ obtaining $R$, a quasi order parameter, and this is plotted in Fig. 2(left). $R$ clearly approaches zero around $T_{c}$ indicating parity doubling. This effect is strongest for baryons with the smallest strangeness content, as expected due to the residual chiral symmetry breaking effects from the strange quark.

We obtain a measure of the transition temperature for each channel using the point of inflection of $R$ and plot these in Fig. 2 (right) together with the $T_{c}$ value obtained from the Polyakov loop [4].

The ground state masses are extracted from the correlators in the hadronic phase using conventional exponential fits with the results displayed in Fig. 3. We see that the $T=0$ masses are heavier than the experimental values - this reflects the non-physical value of the two lightest dynamical quarks in our simulation. The positive parity states' masses appear to be $T$-independent, whereas the negative states' masses decrease as $T \rightarrow T_{c}$.
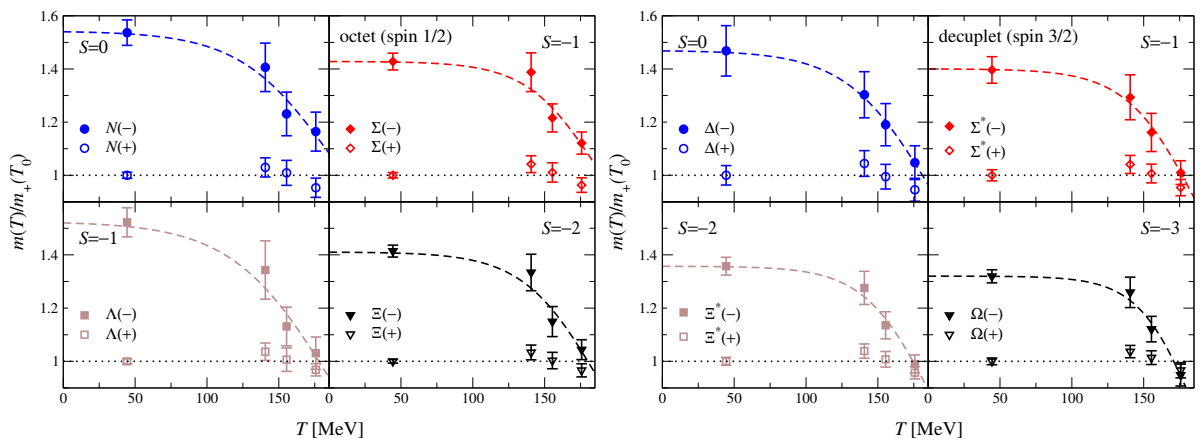

Fig. 3. Temperature dependent masses of the octet and decuplet baryons for both parity sectors.

By using these temperature-dependent masses in the Hadron Resonance Gas (HRG) model, we obtain "in-medium HRG" predictions for the partial pressures and susceptibility $\chi_{B S}=\frac{1}{V T}\langle B S\rangle$, where $B$ and $S$ are the baryon and strangeness number, see Fig. 4 . For $\chi_{B S}$ and the strange partial pressure sectors, this in-medium HRG gives better agreement with independently obtained lattice results [10].

To interpret hadronic correlation functions in the plasma phase, where exponential fits no longer work and we assume there are no bound states, we introduce spectral functions [6],

$$
G_{ \pm}(\tau)=\int_{-\infty}^{\infty} \frac{d \omega}{2 \pi} K(\tau, \omega) \rho_{ \pm}(\omega), \quad \text { where } K(\tau, \omega)=\frac{e^{-\omega \tau}}{1+e^{-\omega / T}} .
$$

We use the Maximum Entropy Method to solve the above inverse problem for $\rho(\omega)$, noting that the positive (negative) parity states appear for $\omega>0(\omega<0)$. 
Spectral function results for a selection of channels are shown in Fig. 5 for a representative temperature in each phase. We see clear ground states in both parity sectors in the hadronic phase, and signs of parity doubling in the plasma phase, particularly for the non-strange baryons.
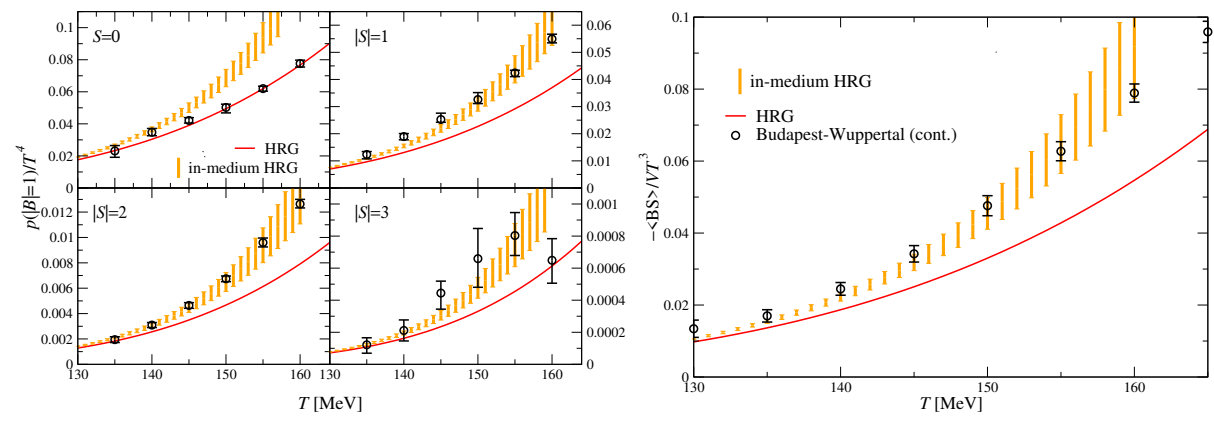

Fig. 4. In-medium Hadron Resonance Gas results. (Left) partial pressures for each strangeness sector, and (Right) the susceptibility $\langle B S\rangle$.
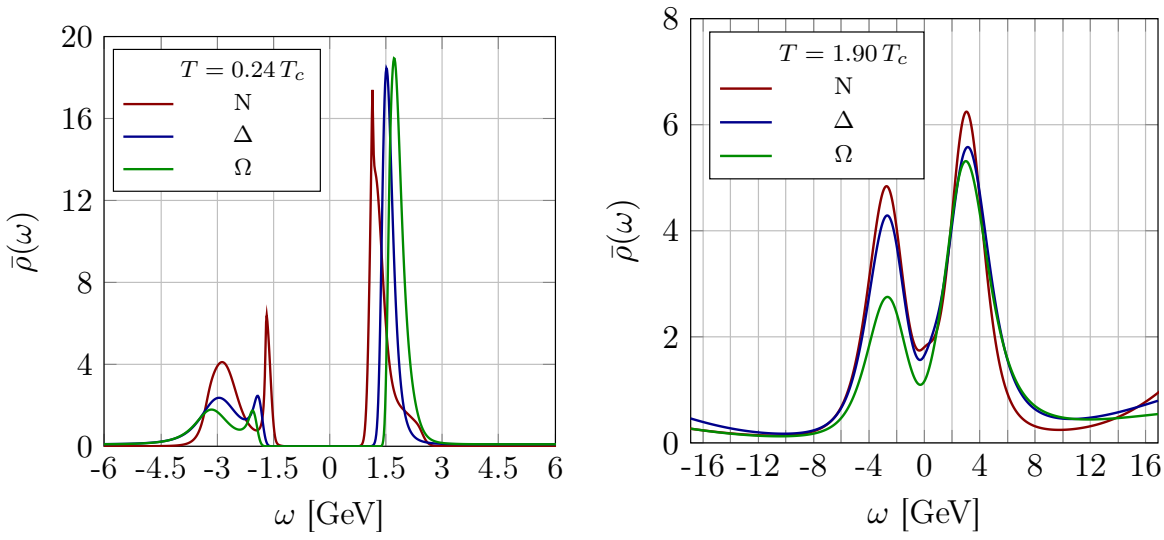

Fig. 5. Spectral function for indicative temperatures in the (left) hadronic and (right) plasma phases for three baryon channels. The details of our Maximum Entropy Method procedure, including choices of default model are detailed in 6 .

\section{Conclusion}

This work uses lattice simulations of $2+1$ flavour QCD to analyse the parity states in the baryonic spectrum as the temperature is varied from essentially 
zero to around $350 \mathrm{MeV}$. Our FASTUM collaboration's lattices are anisotropic which increases the sampling rate in the temporal direction, thereby enhancing the accuracy of our results. We employ a variety of approaches in this work: using the correlation functions directly, conventional fits to exponentials, and extracting the spectral functions using the Maximum Entropy Method.

In the hadronic phase, these methods indicate that the positive parity ground state masses are temperature independent (within errors) and that the negative parity states' masses decrease as $T$ increases until becoming essentially degenerate with its positive parity partner at or close to $T_{c}$. This "parity-doubling" is particularly evident for baryons with the smallest strange content, and this is to be expected due to the strange quark's chiral symmetry breaking effects. This pattern is observed for both octet and decuplet states.

We plan to use both lighter quarks and lattices with finer (temporal) spacings to test the systematics of our approach in our next generation ensembles.

\section{Acknowledgements}

We acknowledge PRACE for awarding us access to Marconi at CINECA, Italy. This work used the STFC DiRAC Blue Gene Q system at the University of Edinburgh, U.K. We have been supported by the STFC grant ST/P00055X/1, and the Swansea Academy for Advanced Computing.

\section{References}

1. C. E. DeTar and J. B. Kogut, Phys. Rev. Lett. 59 (1987) 399; Phys. Rev. D 36 (1987) 2828.

2. I. Pushkina et al. [QCD-TARO Collaboration], Phys. Lett. B 609 (2005) 265 heplat/0410017.

3. S. Datta, S. Gupta, M. Padmanath, J. Maiti and N. Mathur, JHEP 1302 (2013) 145 arXiv:1212.2927 [hep-lat]].

4. G. Aarts, C. Allton, A. Amato, P. Giudice, S. Hands and J. I. Skullerud, JHEP 1502 (2015) 186 doi:10.1007/JHEP02(2015)186 arXiv:1412.6411 [hep-lat]].

5. G. Aarts, C. Allton, S. Hands, B. Jger, C. Praki and J. I. Skullerud, Phys. Rev. D 92 (2015) no.1, 014503 arXiv:1502.03603 [hep-lat]].

6. G. Aarts, C. Allton, D. De Boni, S. Hands, B. Jger, C. Praki and J. I. Skullerud, JHEP 1706 (2017) 034 arXiv:1703.09246 [hep-lat]].

7. G. Aarts, C. Allton, D. De Boni and B. Jger, Phys. Rev. D 99 (2019) no.7, 074503 arXiv:1812.07393 [hep-lat]].

8. R. G. Edwards, B. Joo and H. W. Lin, Phys. Rev. D 78 (2008) 054501 doi:10.1103/PhysRevD.78.054501 arXiv:0803.3960 [hep-lat]].

9. M. Tanabashi et al. [Particle Data Group], Phys. Rev. D 98 (2018) no.3, 030001.

10. S. Borsanyi, Z. Fodor, S. D. Katz, S. Krieg, C. Ratti and K. Szabo, JHEP 1201, 138 (2012) arXiv:1112.4416 [hep-lat]], R. Bellwied, S. Borsanyi, Z. Fodor, S. D. Katz, A. Pasztor, C. Ratti and K. K. Szabo, Phys. Rev. D 92 (2015) no.11, 114505 arXiv:1507.04627 [hep-lat]], P. Alba et al., Phys. Rev. D 96 (2017) no.3, 034517 arXiv:1702.01113 [hep-lat]]. 